\title{
A Hybrid Architecture combining Artificial intelligence and Blockchain for IoT Applications
}

\author{
Dr. D. Sivaganesan \\ Professor, \\ Department of Computer Engineering \\ PSG Institute of Technology and Applied Research \\ Coimbatore, India \\ Email: sivaganesan@psgitech.ac.in
}

\begin{abstract}
As Internet of Things (IoT) revolutionized over the years, it has become a crucial part in many application such as smart city and smart transportation, ensuring smooth functioning of the human life in a more reliable manner. As the amount of data that is to be collected, stored and analyzed increases, there is need for a system that perform these tasks in a more efficient manner. Artificial intelligence plays a crucial part in delivering these requirements. But when AI is involved, it will also result in the use of big tools that are necessary for tackling the issue at hand. Hence in order to ensure that AI can function in a smooth manner, blockchain support is introduced such that it develops a decentralized architecture. Block chain will provide a safe and secure environment to share the information and resources and can also address the drawbacks of Artificial intelligence. The purpose of this proposed work is to develop a hybrid architecture, combining the advantages of blockchain and artificial intelligence to enable big data analysis. We have performed an analysis and results have been observed to conclude the impact of blockchain in Artificial Intelligence in the aspect of latency and accuracy. The results indicate that the proposed architecture excels over the other architectures and can also overcome some of the challenges prevalent.
\end{abstract}

Keywords: Big Data; Internet of Things; Blockchain; Artificial Intelligence; Security

\section{Introduction}

One way of providing connection between the humans and the peripherals is with the help of Internet of Things. They are used to communicate with each other and other devices such as intelligent automotive, smart vehicle, smart homes etc. But, there are a number of discrepancies faced such as GIS visualization, hardware capabilities, centralization, connectivity, security, big data analytics etc. Survey shows that in the year 2014 [1]. Over 750,000 people were subject to spam emails and distribute phishing during the year 2014 alone. In fact, there is much threat in using big data. In order to overcome these issues, a number of technological advancements like Reinforcement Learning and Deep Learning are used in the field of Artificial Intelligence [2]. Deep learning is commonly used for detection, classification, prediction and decision analysis of future movements in IoT. This also enables scaling and feature extraction in data gathered from different sources of IoT. To enhance the efficiency of big data analysis as well as to tackle the length of computation, a number of architecture and framework have been proposed in the recent researches [3-4].

Based on these researches, one can categorize the study into Device analysis, Edge analysis, fog analysis and cloud analysis. Device analysis is used when the devices that are being used are connected to one another [5]. Cloud analysis employs the use of cloud in order to execute and control the operations in the device or system. On the other hand, fog analysis faces severe problem like scalability, resource management etc. These issues can be tackled by edge analysis with the help of an adopted architecture that uses load distribution. The task is completed with the help of cloud server and edge node by task processing. The biggest challenges faced by the already existing methods include centralization, security, privacy, low latency and lack of accuracy. Blockchain serves as an excellent methodology to address these issues. Blockchain technology finds its applications in a number of fields such as healthcare, agriculture, data security and finance [6]. While transacting data, it should be properly secured using digital sign, explainable, secure and immutable.

The major contribution of our proposed work includes:

- Four intelligence such as device intelligence, edge intelligence, fog intelligence and cloud intelligence are incorporated implementing the advantages of Artificial intelligence and Blockchain.

- Issues in blockchain are mitigated using AI and the same goes vice versa.

The paper is organised such that section 2 describes a survey of previous researches followed by proposed methodology [7]. The results simulated are graphically represented and base on the analysis of the results, the 
conclusion for the paper is drawn. The fundamental concept of AI and Blockchain in IoT is represented in the Fig.1.

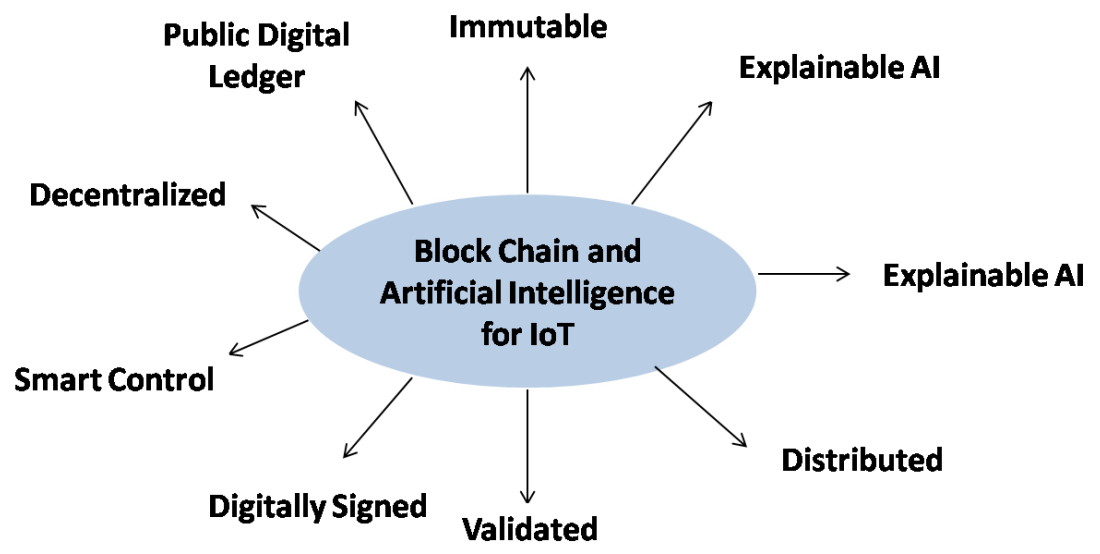

Fig.1. Basic Concept of AI and Blockchain in IoT

\section{Proposed Methodology}

In this paper, we introduce a hybrid architecture that combines the architecture of Artificial Intelligence and blockchain. This architecture is built up of four blocks of intelligence that is used to develop a system that is capable of achieving high level of security with big data analysis and can be used in smart transportation, smart city and smart healthcare. The architecture of the proposed work is represented in Fig.2.

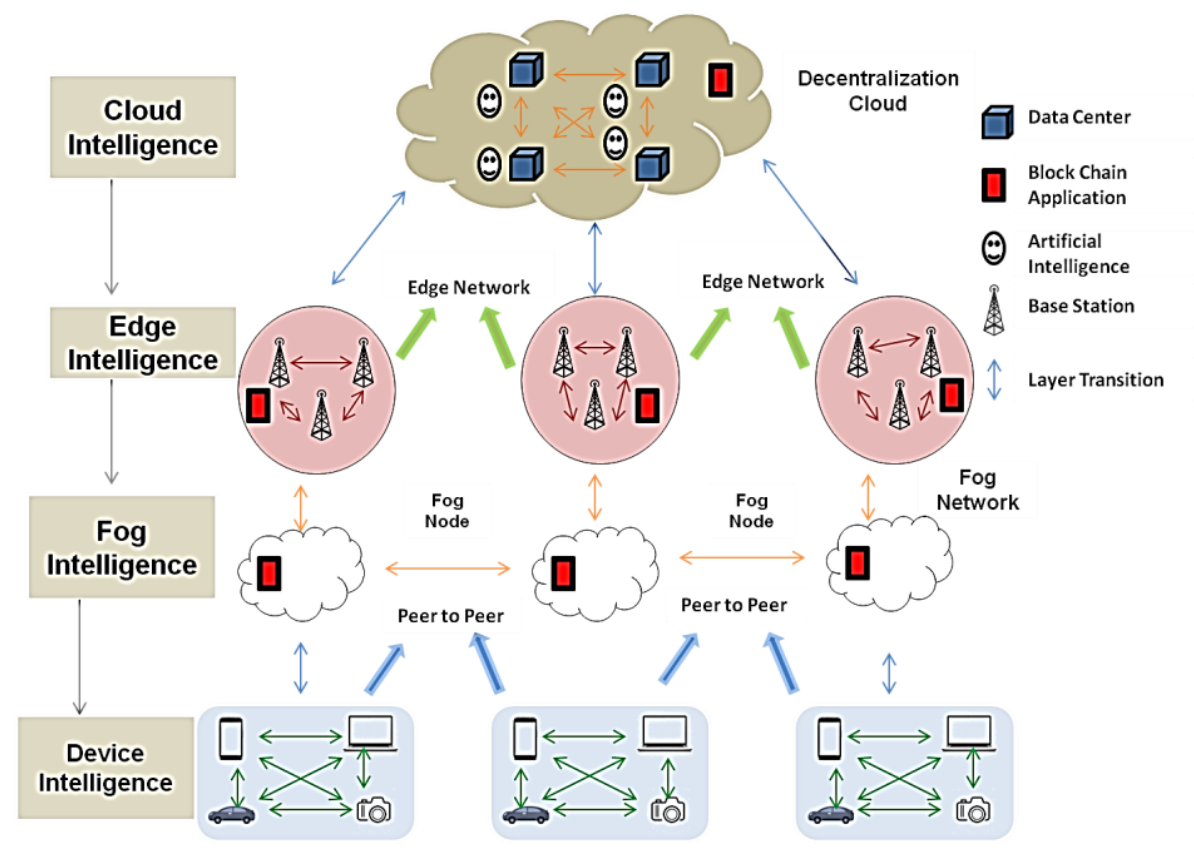

Fig.2. Architecture of Proposed Hybrid System

Blockchain and Artificial intelligence are two fields that have been progressing exponentially over the past decade [8]. A blockchain is made up of four main components: a timestamp, hash value of previous and present block and details of transaction. Each transaction that is made is saved in different nodes such that no other unknown party can access the transaction details or alter it in any away. The hash value is used to ensure security of the data by cryptographic signing which is verified at the receiving end [9]. The data of a ledger can be shared using blockchain in a more trusted manner. Common files can be transferred with the help of IPFS in a secure manner. In [10] different IoT categories have been proposed and how they can be used to create a large set of data. Similarly, a machine that is used to perform a number of operations like problem solving, reasoning, learning and perceiving is called Artificial Intelligence. Novel methods like neural network, deep learning and machine learning are some of the AI techniques that are being used today. These AI machines are being used in many fields like mechanized farming, automated vehicle driving, automatic sensing devices and medical science. Machine learning plays a crucial role in IoT to provide solution to a IoT issues like data prediction, association and analysis. 
The four layers of the architecture are as follows:

- Cloud intelligence is used for integration, analysis and selection of data takes place using centralized methods by the intelligent agents of AI. To ensure secure big data analysis, a distributed pattern is proposed by the blockchain. On request from the scheduler, it can also address issues related energy resource management. AI enabled data centers that are connected to blockchain is present in the cloud in this proposed work which can be used in a number of IoT applications to address a number of issues such as persistence, anonymity, immutability and security.

- At fog intelligence, decision making process takes place in a quick manner with the help of machine learning models that are trained in this layer. A basic set of rules and regulations is followed by the different parties involved during data transferring in the IoT network.

- Edge intelligence uses AI with analytic tools for representation, scaling and feature extraction. It makes use of images and speeches in areas like transportation and healthcare. This layer ensures that a proper understanding between the peer connections is established and offers an efficient and reliable learning task layer.

- Device Intelligence is used for data forwarding and collection with the aid of AI technology. Using deep learning and blockchain, it can be used to enhance privacy and security of the IoT data.

In this work, the IoT platform is built with six layers namely application layer, management layer, service layer, link control layer, communication layer and physical layer. The application layer is associated with cloud intelligence, the management layer and communication layer are linked to fog intelligence while the device intelligence is connected to the physical layer. In the first step, physical information is gathered from the environment with the help of physical layer. This information is further transferred from one system to the other with the use of communication layer as the medium. Security and scalability is enhanced by consensus protocol in management layer. Information can also be stored in the layer and it can be further sent to the service layer where virtual entity resolution, service composition and organization, database support and decision support are incorporated.

Finally, in the application layer, the information is shared such that it can be used in IoT applications. There are two ways in which the proposed architecture can be analyzed namely quantitative analysis and qualitative analysis. Analytics Intelligence is used to collect or generate data with the help of sensing devices from different applications. A unique identity is provided by digital identity with the help of scripting code, hash function and different issuing methods. Distributed cloud storage is part of the proposed methodology wherein it provides the cloud with transparency and traceability such that it can be used in smart contract, microservers and intelligent storage. The centralized server used might attract third party hackers and malicious virus, disrupting the work flow. Hence a decentralized and distributed environment is provided using block chain technology that ensures that the parties involved are safe using proof of authority, proof of stake and proof of work.

\section{Results and Discussion}

Quantitative analysis is observed by the existing researches and a number of standard parameters such as energy cost, computational complexity, security and privacy, latency and accuracy were analyzed in [11-14]. Similarly, a comparative analysis is made on fog, edge and device intelligence used in the proposed hybrid architecture and is represented in Fig.3 and Fig.4. Latency analysis and Accuracy analysis are performed and simulation result shows that the latency value varies from 0 to 12 millisecond using fog intelligence, 0 to 45 millisecond using edge intelligence and 0 to 36 millisecond using device intelligence without using blockchain and 0 to 20 millisecond using fog intelligence, 0 to 55 millisecond using edge intelligence and 0 to 60 millisecond using device intelligence with blockchain. 


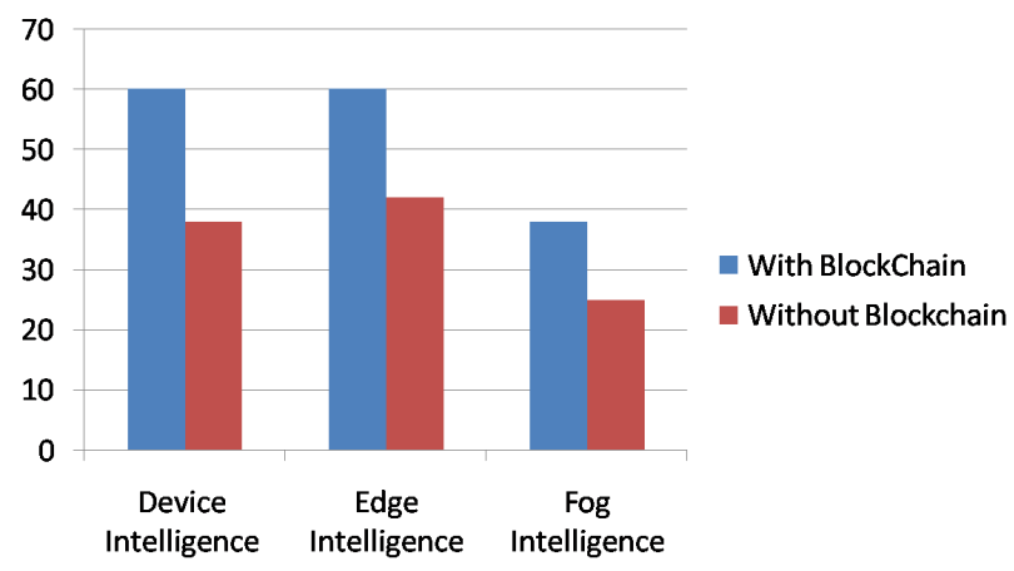

Fig.3. Latency Analysis

Similarly, in accuracy analysis, the accuracy percentage spikes from 0 to $91 \%$ using fog intelligence, 0 to $70 \%$ using edge intelligence and 0 to $69 \%$ using device intelligence, using blockchain. On the other hand, without blockchain, the accuracy percentage is 0 to $82 \%$ in fog intelligence, 0 to $60 \%$ using edge intelligence and 0 to $60 \%$ using device intelligence as observed in Fig.4.

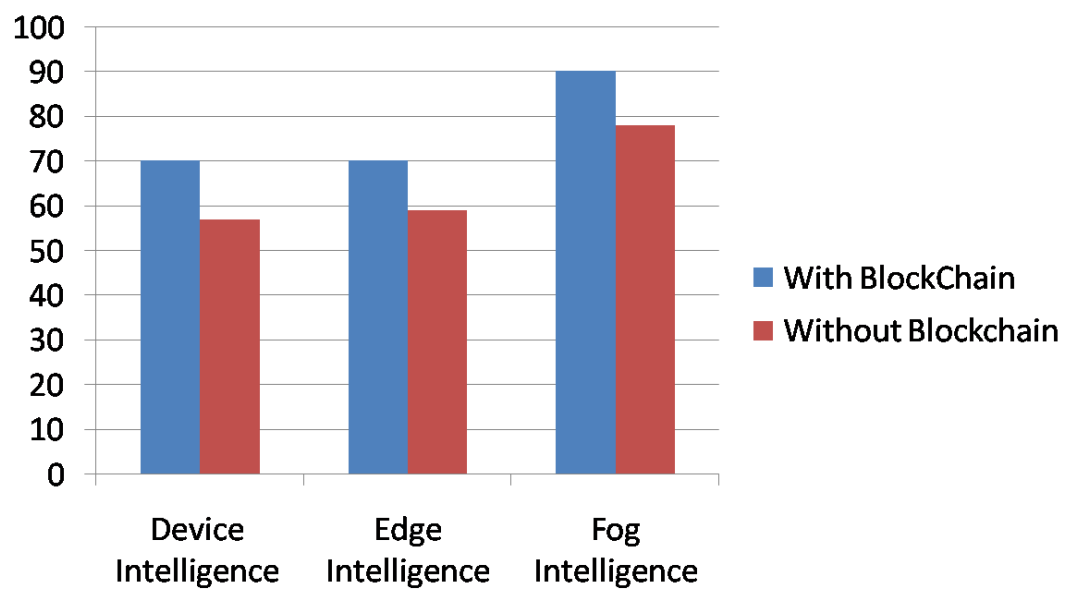

Fig.4. Accuracy Analysis

\section{Conclusion}

In this work we have incorporated a combination of Artificial Intelligence and Block chain in IoT architecture to achieve a device that is embedded with device intelligence, edge intelligence, fog intelligence and cloud intelligence. Smart city, smart healthcare and smart industries are some of the IoT applications that can best use this methodology to ensure secure and scalable output. This work proposes to mitigate a number of issues and challenges in both AI as well as blockchain by combining the two methodologies. Based on the simulation results it is found that we have achieved high accuracy with acceptable latency as well as security using decentralized methodology. Our methodology addresses some research challenges like energy efficiency, cost and capability constraints, heterogeneity, integrity of data flow, anonymity, lack of standard, resource management, interoperability and scalability enhancement. In the future, this hybrid architecture can be enhanced by incorporating machine intelligence methods like classification, scaling and feature extraction. The important challenges faced in the proposed methodology include adoption of the techniques which proves to be a difficult task. To merge AI with blockchain, a number of architectures and prevention mechanism can also be introduced as a future direction. In large IoT application scenario, the issues of traffic monitoring and address space solution can also be explored with more technological inventions.

\section{References}

[1] Salah, K., Rehman, M. H. U., Nizamuddin, N., \& Al-Fuqaha, A. (2019). Blockchain for AI: Review and open research challenges. IEEE Access, 7, 10127-10149. 
[2] El Azzaoui, A., Singh, S. K., Pan, Y., \& Park, J. H. (2020). Block5gintell: blockchain for ai-enabled 5G networks. IEEE Access, 8, 145918-145935.

[3] Shirley, D. R. A., Ranjani, K., Arunachalam, G., \& Janeera, D. A. (2020). Automatic Distributed Gardening System Using Object Recognition and Visual Servoing. In Inventive Communication and Computational Technologies (pp. 359-369). Springer, Singapore.

[4] Kumar, R., Wang, W., Kumar, J., Yang, T., Khan, A., Ali, W., \& Ali, I. (2020). An Integration of blockchain and AI for secure data sharing and detection of CT images for the hospitals. Computerized Medical Imaging and Graphics, 87, 101812.

[5] Wang, K., Dong, J., Wang, Y., \& Yin, H. (2019). Securing data with blockchain and AI. IEEE Access, 7, 77981-77989.

[6] Pan, Q., Wu, J., Li, J., Yang, W., \& Guan, Z. (2020). Blockchain and AI Empowered Trust-InformationCentric Network for Beyond 5G. IEEE Network, 34(6), 38-45.

[7] Sri, P. D., Luharuka, S., Somani, R., \& Kulkarni, G. A. Technical Scrutiny of Block chain Technology Protocols and its Applications.

[8] Wang, T., Liew, S. C., \& Zhang, S. (2019). When blockchain meets AI: Optimal mining strategy achieved by machine learning. arXiv preprint arXiv:1911.12942.

[9] Nguyen, D., Ding, M., Pathirana, P. N., \& Seneviratne, A. (2020). Blockchain and AI-based Solutions to Combat Coronavirus (COVID-19)-like Epidemics: A Survey.

[10] Gulati, P., Sharma, A., Bhasin, K., \& Azad, C. (2020). Approaches of Blockchain with AI: Challenges \& Future Direction. Available at SSRN 3600735.

[11] Michailidis, M. P. (2018). Hie Challenges of AI and Blockchain on HR Recruiting Practices. Cyprus Review, 30(2).

[12] Rabah, K. (2018). Convergence of AI, IoT, big data and blockchain: a review. The Lake Institute Journal, 1(1), 1-18.

[13] Shae, Z., \& Tsai, J. (2019, July). AI blockchain platform for trusting news. In 2019 IEEE 39th International Conference on Distributed Computing Systemillisecond(ICDCS) (pp. 1610-1619). IEEE.

[14] Sivaganesan, D. (2019). DESIGN AND DEVELOPMENT AI-ENABLED EDGE COMPUTING FOR INTELLIGENT-IOT APPLICATIONS. Journal of trends in Computer Science and Smart technology (TCSST), 1(02), 84-94. 